\title{
Wolf-Rayet features in active galactic nuclei
}

\author{
Anabela C. Gonçalves ${ }^{1,2} \dagger$, \\ M. Serote $\operatorname{Roos}^{1}$ and T. Contini ${ }^{3}$
}

${ }^{1}$ Centro de Astronomia e Astrofísica da U. de Lisboa, Observatório Astronómico de Lisboa, Portugal email: adarbon@oal.ul.pt, serote@oal.ul.pt

${ }^{2}$ LUTH, Observatoire de Paris-Meudon, France email: anabela.darbon@obspm.fr

${ }^{3}$ LA2T, Observatoire Midi-Pyrénnées, Toulouse, France email: contini@ast.obs-mip.fr

\begin{abstract}
It was suggested by Terlevich \& Melnick (1985) that many nuclei classified as Seyfert $2 \mathrm{~s}$ could be associated with WR stars recently formed in a powerful starburst. Since then, the signature of WR stars has been claimed to be observed in several Seyfert $2 \mathrm{~s}$ and LINERs. Gonçalves et al. (1999) have shown that a small number of Seyfert 2 s have very weak [N II] 6548,6584 lines, suggesting that these objects could be partly ionized by WR stars. We have obtained high S/N spectra of such "Weak-[N II] Seyferts" to investigate if WR stars can be observed in galaxies without any other evidence for a starburst nebulosity. Amongst other findings, we have detected the presence of emission features such as $\mathrm{Si}$ II 5056,6347 and Ni II 7380. Other lines, such as [Ar IV] 4711,4740 and [Fe III] 4658 are possibly present, mimicking the Bowen blend.

The detection of WR stars in AGN poses interesting questions concerning the link between nuclear activity and star formation, providing another angle to the study of the Starburst-AGN connection. However, one must be very careful in interpreting the available data; for the time being, the role played by WR stars in these AGN remains an open debate.
\end{abstract}

\section{Introduction}

Weak-[N II] Seyfert 2s (Gonçalves et al. 1999) represent a small subset of narrow emission-line galaxies with weak $[\mathrm{N} \mathrm{II}] 6584 / \mathrm{H} \alpha$ line ratios $(<0.4)$; the relative strength of emission lines such as [O III] 4959,5007, [S II] 6717,6731 or [O I] 6300,6363 is, however, typical of Seyferts. Weak-[N II] Seyfert 2s do not seem to be "composite" objects, which are lying at intermediate positions in classical optical diagnostic diagrams (Véron et al. 1997; Gonçalves et al. 1999). Their location on the $\log ([\mathrm{O} \mathrm{III}] / \mathrm{H} \beta)$ vs. $\log ([\mathrm{N} \mathrm{II}] / \mathrm{H} \alpha)$ diagram (see Fig. 1, on the left-hand side) is found to overlap with the region occupied by nebulae ionized by Wolf-Rayet (WR) stars, suggesting that these could contribute to, or even dominate, the energetics of Weak-[N II] Seyferts.

WR galaxies (WRGs) are starburst systems in which the presence of WR stars can be inferred. WR features can be observed as a "bump" at $4686 \AA$ (corresponding to the He II line) and/or faint emission corresponding to the N III 4640, C IV 5808 and C III 5695 lines. Most WRGs are "classical" star-forming galaxies; only a few are known to harbour an active nucleus, e.g. NGC 6764 and Mrk 309 (Osterbrock \& Cohen 1982), Mrk 477 (Heckman et al. 1997), or Mrk 1210 (Storchi-Bergmann et al. 1998).

Weak-[N II] Seyfert 2s seem to constitute excellent WRGs candidates and powerful tools in the study of the Starburst-AGN connection. However, can WR stars be truly observed in galaxies with "pure" Seyfert spectra, without any other evidence for a starburst nebulosity?

$\dagger$ Send offprint requests to A. C. Gonçalves: adarbon@oal.ul.pt or anabela.darbon@obspm.fr 

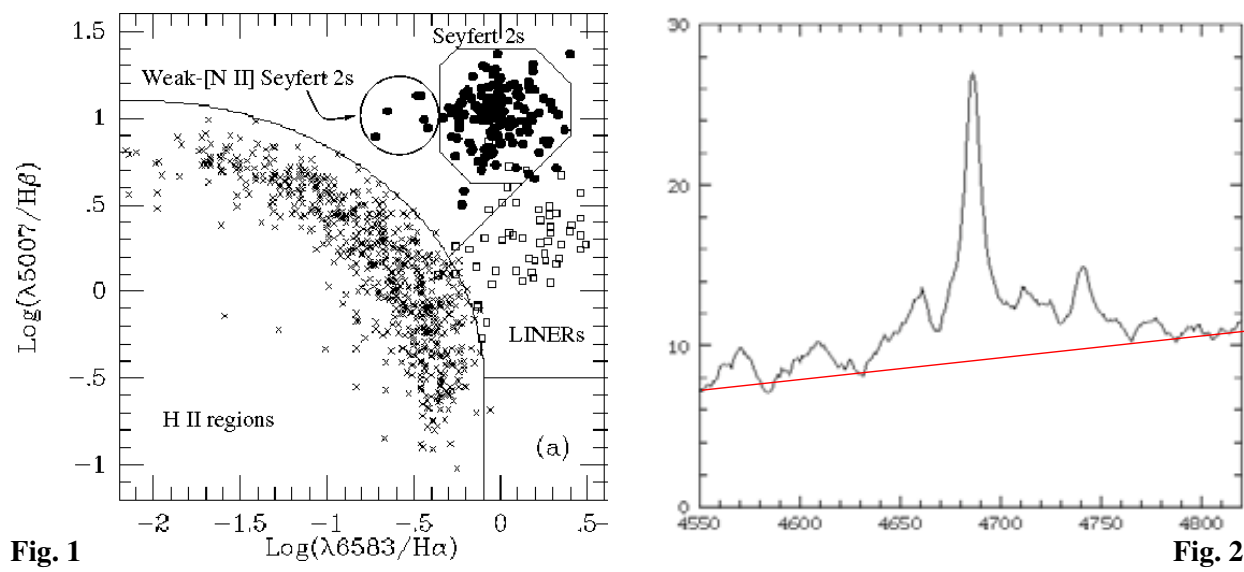

Fig. 1

\section{Observations and data reduction}

With these questions in mind, we have obtained long-slit spectra with EMMI at the ESO/NTT; the slit-width was $1^{\prime \prime} .0$ and the resolution was $\sim 5 \AA(F W H M)$. The sample consisted of 2 known AGNs with WR features, 4 weak-[N II] Seyfert 2s, 1 WRG template and 1 "classical" Seyfert 2. The nuclear stellar contribution was removed by using galaxy templates and the iron contamination was subtracted from the data by using a suitable fraction of a template based on I Zw 1. The emission line spectra were fitted in terms of Gaussian profiles (a full description of the method is given in Gonçalves et al. 1999).

\section{Preliminary results and ongoing work}

A plethora of forbidden emission lines ([Ar IV] 4711,4740, [Ne IV] 4714,4716,4724,4726), permitted lines (Si II 5056,6347, Ni II 7380) and iron features (e.g. [Fe III] 4658,4702, Fe II blends and fluorescent Fe I lines) are possibly present in the spectra. Some of these lines can successfully mimic a Bowen blend, suggesting the presence of WR stars (Fig. 2, on the right-hand side, display features often interpreted as a WR bump); one must therefore be very careful in the analysis and interpretation of the data. In addition, whenever WR features are identified, they seem to be accompanied by a starburst component, the spectrum having, therefore, a "composite" nature; this suggests that the WR stars are located within the $\mathrm{H}$ II region and have no direct connection with the AGN.

Further analysis of the data relying on a robust stellar population synthesis and on a more accurate iron correction (see Véron-Cetty et al. 2004 and Joly et al. in these proceedings) is under way; such a study will allow us to conclude on the presence of WR stars in these active nuclei, with implications to the Starburst-AGN connection debate.

\section{Acknowledgements}

A. C. G. and M. S. R. acknowledge support from the portuguese Fundação para a Ciência e a Tecnologia through grants no. BPD/9422/2002 and BPD/5684/2001, respectively.

\section{References}

Gonçalves, A. C., Véron-Cetty, M.-P., \& Véron, P. 1999, A\&AS, 135, 437

Heckman, T. M, et al. 1997, ApJ, 482, 114

Osterbrock, D. E., \& Cohen, R. D. 1982, ApJ, 261, 64

Storchi-Bergmann, T., Fernandes, R. C., \& Schmitt, H. R. 1998, ApJ, 501, 94

Terlevich, R., \& Melnick, J. 1985, MNRAS, 213, 841

Véron, P., Gonçalves, A. C., \& Véron-Cetty, M.-P. 1997, A\&A, 319, 52

Véron-Cetty, M.-P., Joly, M., \& Véron, P. 2004, A\&A, 417, 515 